\title{
Medicine Delivering and Patient Parameter Monitoring Robot - MedRobo
}

\author{
Abhilash Joy ${ }^{1}$, Rojin Anto Varghese ${ }^{2}$, Angela Varghese ${ }^{3}$, \\ Anna Mary Sajeev ${ }^{4}$, Mrs. Supriya Mary Sunil ${ }^{5}$ \\ 1,2,3,4 UG - Biomedical Engineering, Sahrdaya College of Engineering and Technology, Thrissur, Kerala \\ ${ }^{5}$ Assistant Professor, Biomedical Engineering, Sahrdaya College of Engineering and Technology, Thrissur, Kerala
}

\begin{abstract}
In this paper, we propose a robot named MedRobo with some functionality of providing medicine as well as to measure the vital parameters (Heart rate, Pressure, Temperature and $\mathrm{SpO} 2$ ) of the patient. During the current scenario, reducing the human to human contact in hospitals is required. In a bid to prevent doctors and medical staff from getting affected by Coronavirus, the role of medicine delivering robots is evolving. We can attain the locomotion procedure of the robot using the principle of Radio-frequency identification (RFID) that automatically identifies and tracks tags attached to the objects. The movement and finding the path to patient location is done through a line follower and with RFID tag. Line following method is used to identify the path with help of two infrared sensors. The robot will move towards the patient's room by following a non reflective line and use RFID cards to identify the patient's room number. Using the vending machine the medicine delivery is made possible to the patients. All the measured parameters will be stored to the cloud using the application of the Internet of Thinking (IoT). All the data will successfully be sent to the cloud through the Node MCU. If the read values varied from threshold then a message will be sent to doctors through GSM Module.
\end{abstract}

Keywords: Line following Robots, RFID, IoT, NodeMCU, GSM Module.

\section{INTRODUCTION}

Nowadays Robotics is a wide spreading area almost in all fields including healthcare. In healthcare applications, IoT robots are involved for surgery, care taking, motion robots and medication robots [1-3]. Robotic systems have dramatically extended the reach of humans in sensing, interacting, manipulating and remodelling the world around us [4-6]. Medical robotic devices are designed for entirely different environments and operations relevant to the treatment and prevention of diseases. Under this severe pandemic cause of novel coronavirus or covid-19, hospitals are overcrowded due to an increase in lots of new patients. Hence the hospital staff are in a great need of support from technology [7-13]. Robotic healthcare thus finds the best way to support them by providing their superior work and helping them not to have direct contact with the coronavirus positive patients. The important task of a nurse is to take care of patients allotted to them by giving them medicines in proper intervals and recording the patient's body parameters. Delivering the medicine and checking vitals (Heart rate, Pressure, Temperature and SpO2) of the patient in one ward on a daily basis would take around half to one hour. So to reduce the workload of medical staff, the medicine vending and parameter monitoring can be automated. This was the motivation behind developing this project. The rest of the paper is organized as follows, in section 2 discuss the existing methods available in hospitals and their drawback, section 3 explanation of proposed model with block diagram. And in the end result \& conclusion in section 4.

\section{EXISTING METHOD AND ITS DRAWBACK}

The existing medicine delivery robots intimate patients to take medicine and deliver pills based on doctor's prescription. These medicine delivering robots help the patients to take the medicine at the correct time alternating nursing services. The nursing robot finds the trail employing a line following technique that identifies the track with the assistance of two infrared proximity sensors and by using RFID cards identifies the area range of the patient. Robot senses a dark colored line by using Infrared sensors and sends a signal to Atmega 2560. The L293D motor driver drives the motor according to sensor outputs. It additionally monitors the pressure and temperature levels of the patient and records it within the hospital patient information by incorporating pressure and digital temperature detector in it. This study declares the introduction of medical robots considerably increased safety and quality of health management systems in comparison to manual systems [9-10]. Zening Shao and his team make up a cloud and IoT Assisted indoor robot for the medicine delivery supported multi core embedded system, RFID, IEEE802.11 communication protocol, and cloud technology. It has an overseas mobile terminal and laptop terminal management system. when the mechanism moves to the patient 
Vol. 8, Issue 6, June 2021

DOI: $10.17148 /$ IARJSET.2021.8679

space, the system can browse the data like quantity of medication from IC card. the system features a fastened threshold worth. If the worth is on top of or equal threshold, the moving mechanism can stop the current position to end reading data and grouping pictures and delivering drugs. System continually uploads patients knowledge and stores it in cloud [11-13]. Nimit Sheth and Sahil Jethwa proposed an internet enabled self-regulating "MEDICAL ASSISTANT" Robot which will interact with patients and deliver medications at regular times acting as a substitute nurse. This robot is an IoT enabled system with features such as lifting arms to carry objects and food packages, delivering medicines, and interacting with patients to overcome feelings of loneliness [14- 20].

The existing medicine delivery robots have some drawbacks. The nurses or doctors do not receive any information whether the medicine is delivered or not. Nurses will have to administer medication to patients and also check their vitals. But in the current scenario the medical staff find it very difficult to regularly expose the patients with coronavirus positive. Under these circumstances the introduction of a robot with the basic functions as that of a hospital staff will be thoughtful and supportive for them.

\section{PROPOSED MODEL}

Our project MedRobo is an alternate solution to the difficulties faced by the hospital staff in treating the coronavirus positive patients, who raised this problem. It delivers the medicine to the patient and checks the important parameters of the patient such as temperature, heart rate, $\mathrm{SpO} 2$ and blood pressure without the involvement of humans or by avoiding the direct contact of hospital staff with the patients. By using the reference parameters which are given to the system ,it will compare with the measured parameters. Then the recorded parameters data will be sent to the doctors through the IOT platform. The movement and finding the path to patient location is done through a line follower and with RFID tag. The medical staff will be aware whether the medicine is delivered or not. Also can reevaluate and view the datas of individual patients after a particular interval of time to make sure that all those patients suffering from coronavirus are feeling better and are in good condition.

The proposed robot, MedRobo mainly consist of 5 parts:

1. Line following robot

2. Medicine vending

3. Parameter monitoring

4. Read values sent to NodeMCU and upload to cloud

5. Message sent to doctors through the GSM module.

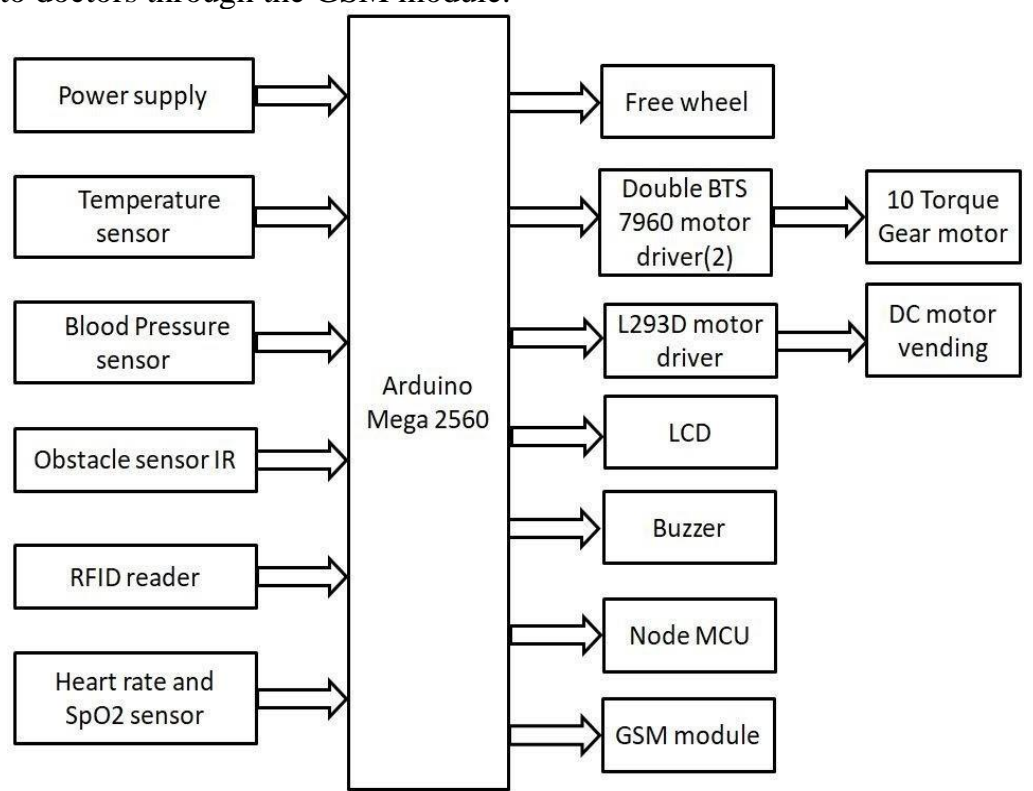

Fig. 1 Block Diagram of Proposed Model

The main block diagram of the MedRobo consists of an arduino mega 2560 as the central part. All the other peripherals for the delivering and parameter measurements are connected to this arduino mega. The temperature sensor, blood pressure sensor and heart and $\mathrm{SpO} 2$ sensors are connected for parameter measurement. For obstacle detection, obstacle sensor IR is used. RFID reader for the path recognition, node mcu and GSM module for the transferring of measured parameters value are connected. Motor drivers are connected for the locomotion and vending purpose namely double BTS 7960 and L293D. The power supply and buzzer are also provided for the robot functioning and indication is 


\section{International Advanced Research Journal in Science, Engineering and Technology}

Vol. 8, Issue 6, June 2021

DOI: $10.17148 /$ IARJSET.2021.8679

shown in above Fig. 1.

\subsection{Pre-processing Stage}

Initially the nurse in the nursing room fills the medicines in the vending machine in order of room.

\subsection{Processing Stage}

The robot starts to move by the line follower in that we use the IR transmitter and receiver. The robot moves by a 12 volt Gear motor and it is controlled by the Double BTS7960 motor driver.

The line following robot follows a line and it can detect this coloured line with the help of IR proximity sensors and send signals to Arduino mega 2560.This IR sensor works like a diode, it consists of a transmitter and receiver. The transmitter transmits the light and the receiver receives it. When the robot changes position from the line the transmitted light is not received by the receiver. Then the motor will stop. In that condition while the receiver is not receiving then the motor moves two steps to right or to left to get back to the line while when the receiver receives the light then again moves. This line following is accomplished by using the IR proximity sensor which is connected to the arduino mega 2560. The Gear motors are driven by the double BTS 7960 motor driver as shown in Fig. 2.

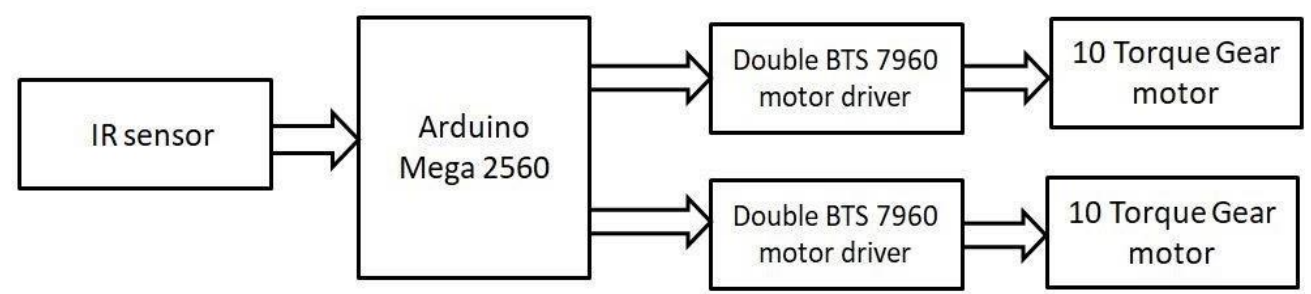

Fig. 2 Block Diagram of Line Following

When it reaches in front of the patient room RFID is detected. The robot will make a buzzer to make attention inside the room and to open the room. When the room opens the robot will move inside the room and reach near to the patient bed. When it reaches the bed it displays the question on the display to start and yes it to process.

First medicine vending occurs. Before starting the process, the user is being asked to confirm vending. If yes, medicine starts vending. This is done by the de motor with motor driver 1293d. Vended medicine falls into a box placed, it consists of an IR sensor while medicine falls into it then IR light cut then vending motor stops and vending also stops. After confirmation, the process of vital patient monitoring starts. The parameters monitoring are temperature, pressure, heart rate and $\mathrm{SpO} 2$. The temperature is measured using LM35 temperature sensor and digital blood pressure sensor is used to measure pressure. MAX30100 sensor module is used to measure both heart rate and SpO2. For this process, initially in the LCD display the message is displayed asking the user to place his/her hand and press confirm. The three parameters temperature, $\mathrm{SpO} 2$ and heart rate is measured and the user is asked to confirm and to take off his/her hands. After this ,it is displayed to put a bp cuff and confirm. After reading the blood pressure, the patient is asked to remove the cuff and to press confirm.

All the values read are sent to NodeMCU which is a microcontroller having an inbuilt wifi driver. It is configured with local wifi. An IP address is provided and the patient ID and parameters are uploaded to this address. In NodeMCU the receiver also has this ID and it checks the ID and compares. After comparing if the ID matches, the read parameters are stored in an array and upload in the corresponding folder. The read values are compared using a threshold and if the value varies from threshold a message is sent to the doctor through the GSM module.

After the process, the patient is asked to confirm the finish. If yes, then the robot will get back to the main floor and move forward to the next room. After the processes, the robot gets disinfected by uv rays before it comes in contact with the next patient. After completing all rooms, the robot gets back to the staffing room. 


\section{International Advanced Research Journal in Science, Engineering and Technology}

Vol. 8, Issue 6, June 2021

DOI: $10.17148 /$ IARJSET.2021.8679

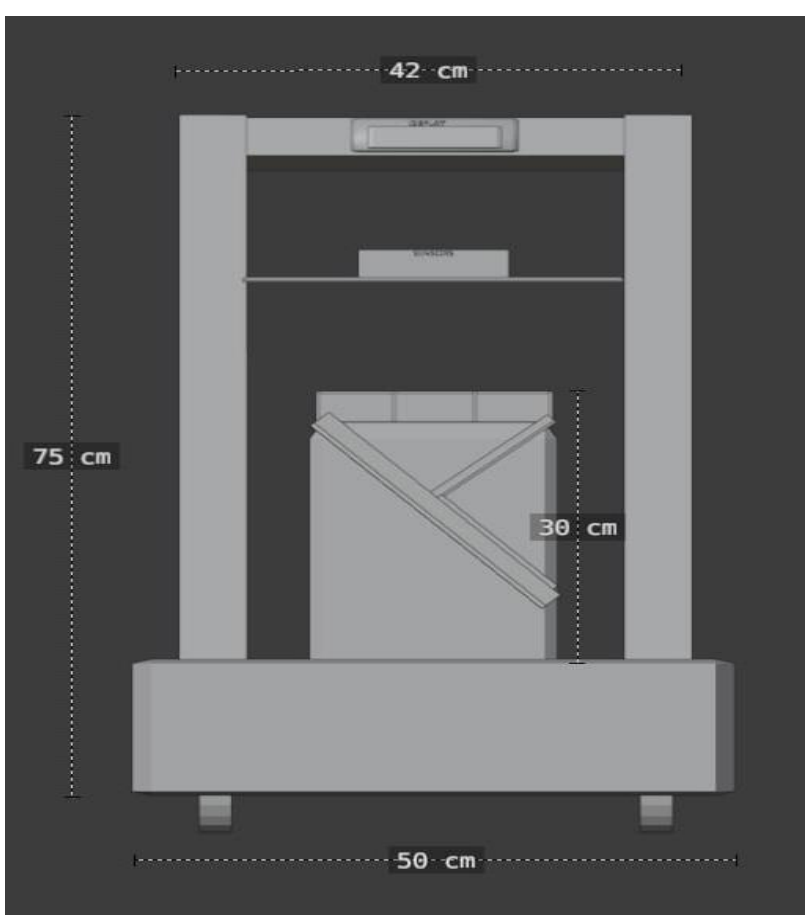

Fig.3 Product Design

\section{RESULTS AND DISCUSSION}

We initially tested the features mainly the robot movement, medicine vending and patient parameter monitoring by simulating the operations in proteus simulation software. The stimulation circuit of robot movement is shown in the below Fig. 4.

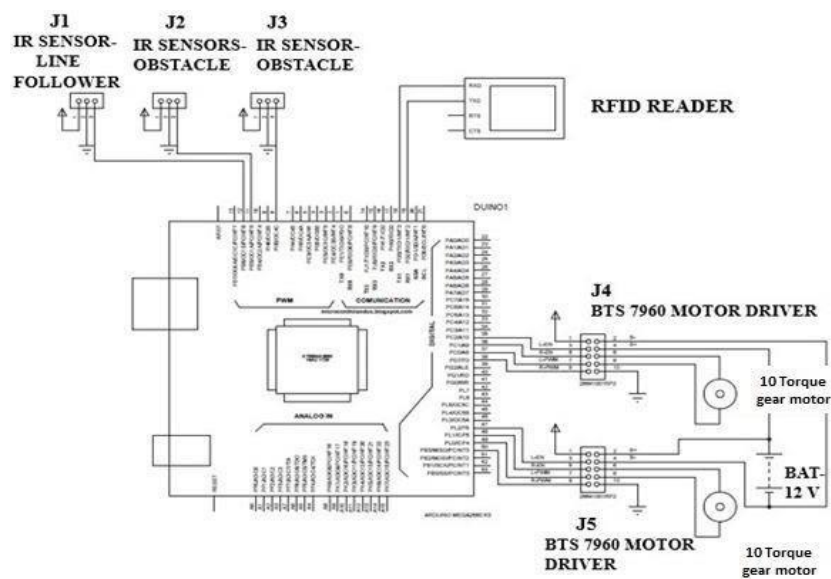

Fig.4 Circuit Diagram of Robot Movement

Here we use Arduino mega 2560. Initially the $12 \mathrm{~V}$ is given to the robot and it starts from sensing the non reflective line drawn on the floor by using an IR sensor J1. Two obstacle IR sensors J2 and J3 are used to detect the presence of obstacles. The robot will start moving towards the patient's room by following the non reflective line and with the help of RFID reader. Robot identifies the patient room. Then the motor stops rotation and it turns to the patient room. The J4 and J5 are the BTS 7960 motor driver and corresponding two Gear motors are used to control the wheel directions. 
Vol. 8, Issue 6, June 2021

DOI: $10.17148 /$ IARJSET.2021.8679

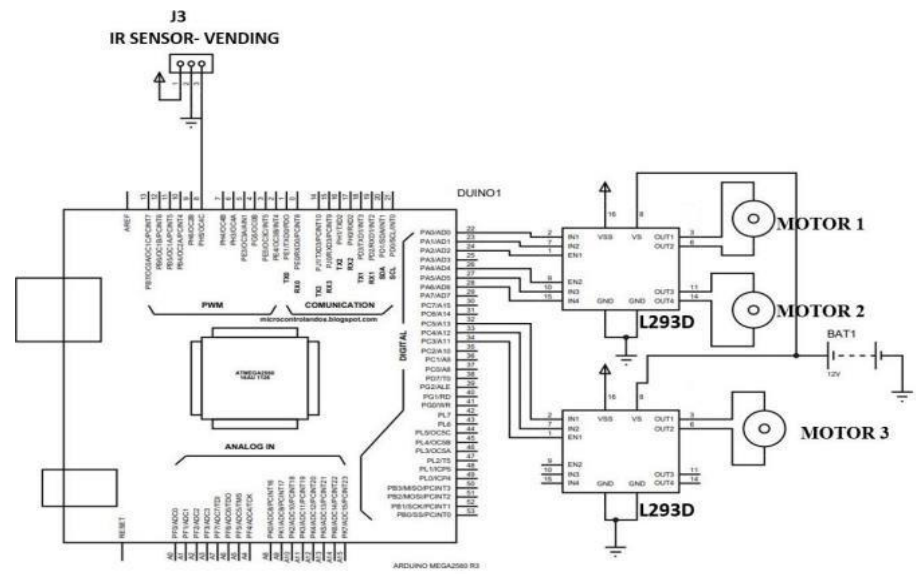

Fig. 5 Medicine Vending Circuit Diagram

When the patient is being asked to confirm for medicine vending the dc vending motor rotates. It consists of three DC motors and is controlled by an L293D motor driver. One motor driver is used to run the two DC motors. Motor U1 moves in forward direction and vended medicine falls into a box placed which consists of an IR sensor (J3). While medicine falls into it then the IR light cuts and then the vending motor stops. After collecting the medicines, the patient is asked to confirm to finish vending. The simulation circuit is shown in Fig. $5 \&$ the hardware setup is shown in Fig. 6.

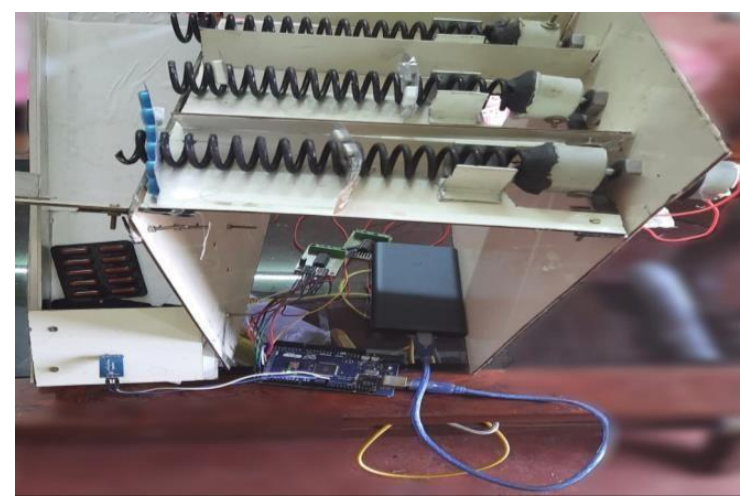

Fig. 6 Hardware setup of Medicine Vending

After the medicine vending, the next step is the patient parameters monitoring. The simulation circuit is shown in the below Fig. 7. Here we check the basic four patient vitals namely heart rate, pressure, temperature and SpO2.Three sensors are used for the measurement. MAX30100 (J1) is used for monitoring heart rate and SpO2 and LM35 (U1) temperature sensors are used for the temperature measurement. Digital blood pressure sensor is used for pressure measurement. The read values are sent to NodeMCU. There it is compared with the stored ID and if it matches, the patient ID and corresponding measured parameters are stored in an array.

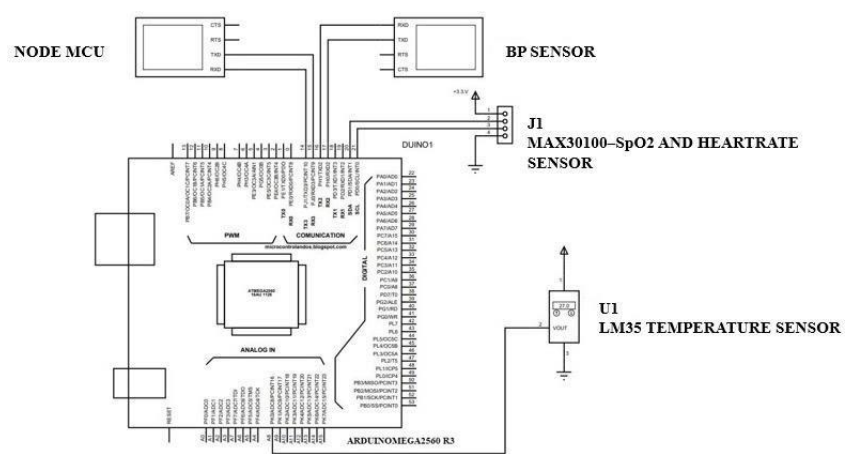

Fig. 7 Parameter Monitoring and IOT module Circuit Diagram

The read values are compared using a threshold and if the value varies from threshold a message is sent to the doctor through the GSM module and the hardware setup of sensors and GSM module shown in fig 8,9. 


\section{International Advanced Research Journal in Science, Engineering and Technology}

Vol. 8, Issue 6, June 2021

DOI: $10.17148 /$ IARJSET.2021.8679

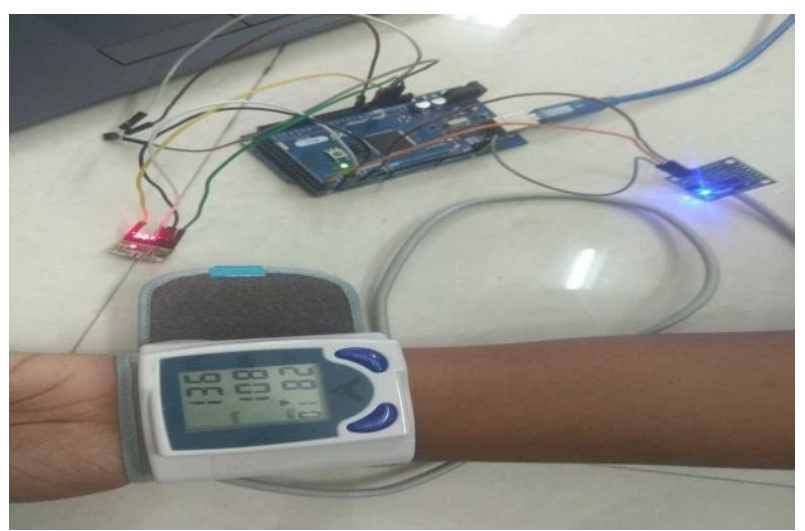

Fig 8 Hardware setup of sensors
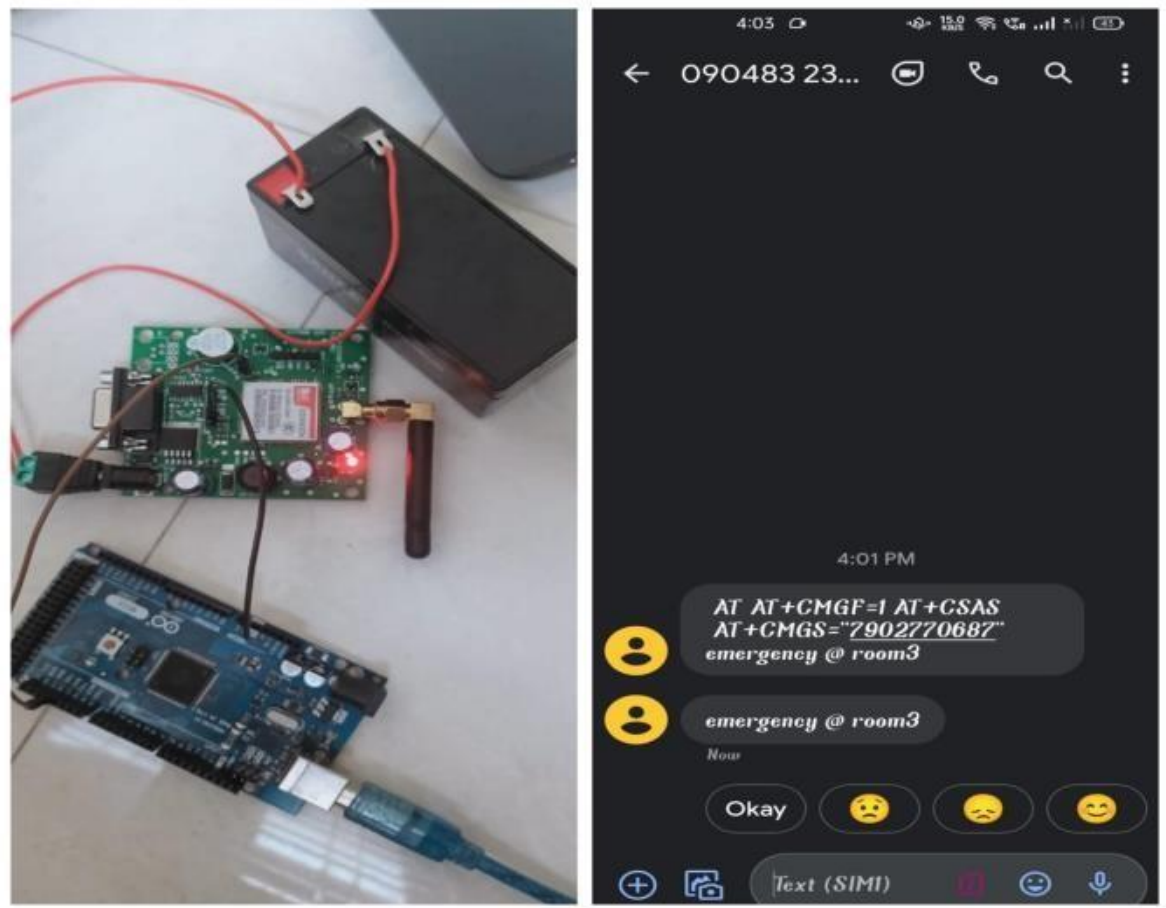

Fig 9 Hardware setup of GSM module

After the process, the patient is asked to confirm to finish. The robot moves backward and moves to the next room and the same process continues. After completing all the rooms, move to the nursing room. The robot is able to disinfect itself after covering all the processes to be carried out in a room. Before it reaches the next room, by using UV rays, the parts that come in contact with the patients were able to be disinfected. The rays are produced as it moves out of the room and covers all those parts without spreading out anywhere. This also does not come in contact with any other objects or humans. Hence it helps in the safe and secure disinfection. 


\section{International Advanced Research Journal in Science, Engineering and Technology}

Vol. 8, Issue 6, June 2021

DOI: $10.17148 /$ IARJSET.2021.8679

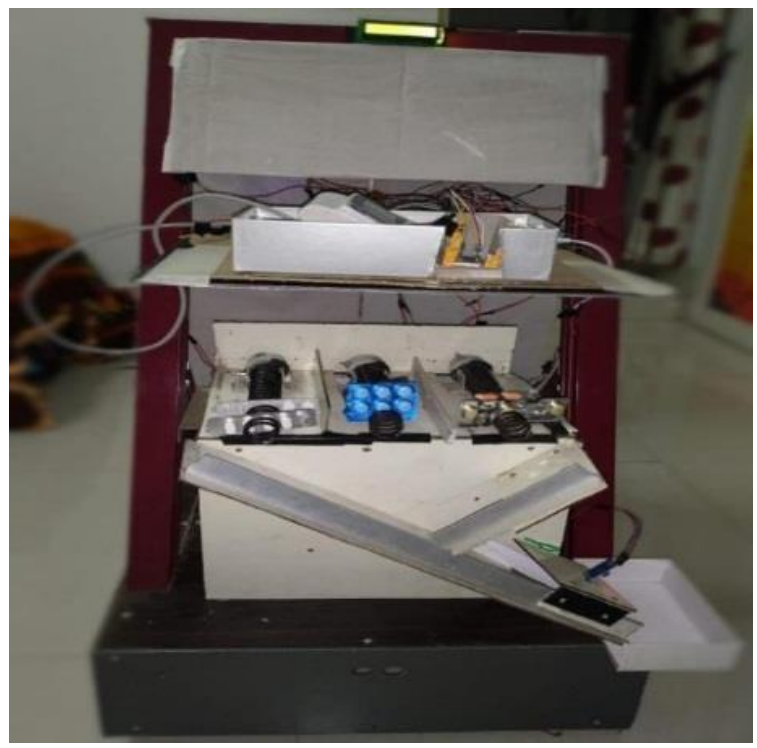

Fig 10 Hardware Setup of MedRobo

\subsection{Stability Check}

The robot is held on a rigid metal frame with three wheels. The possibility of falling is very minute. The two wheels at the back side are connected to the motor which drives the whole robot. One free wheel is attached to the front side which provides good stability to the whole model.The overall stability of the model is calculated by taking the wings of the individual units and parts we took for the development of the robot. First we calculated the weight of the frame in which all the units have to be placed. and it was around $6.105 \mathrm{~kg}$. Then we calculated the weights of all the units and things which we place on that frame like the sensors, weirs, boxes, motors, drivers, batteries etc. it was approximately $2.5 \mathrm{~kg}$. As these weights are less than the weight of the frame, we can say that the overall framework is in a stable condition.

\subsection{Testing and Validation}

We tested the robots with a person, to check the parameters. As the medicine vending is done properly without any interruption, we decided to do the test for parameter monitoring and its further procedures. First we choose a person of age 22, and ask the person to do parameter monitoring. The temperature and heart rate are measured at first and the values are stored. Then the pressure sensor is worn on the wrist, which shows the values of systolic blood pressure, diastolic blood pressure and SpO2. All these values are stored in the cloud using IOT. As the person in which we did the test was healthy and showed only normal variations, the stored values and graphs of the patient using ThingSpeak are shown below:

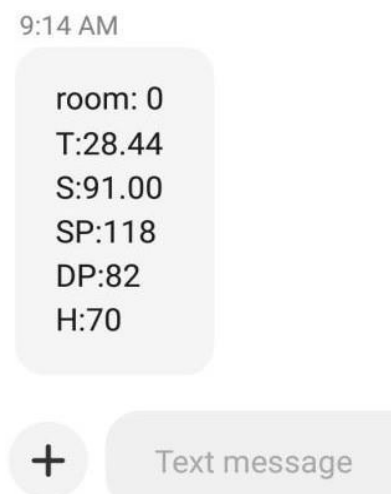

Fig 10. The measured values as a message 


\section{International Advanced Research Journal in Science, Engineering and Technology}

Vol. 8, Issue 6, June 2021

DOI: 10.17148/IARJSET.2021.8679

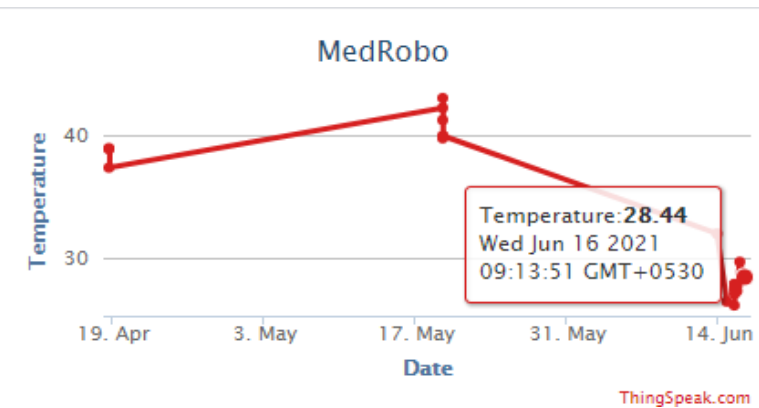

Fig 11. Parameter chart of Temperature

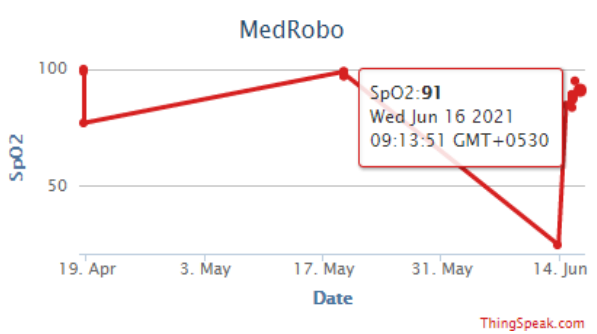

Fig 12. Parameter Chart of SpO2

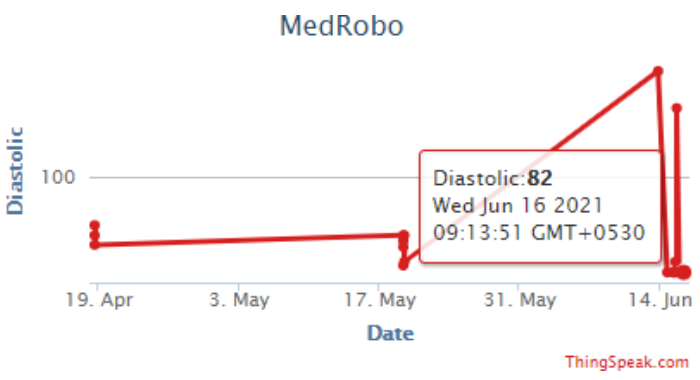

Fig 14. Parameter chart of Diastolic blood pressure

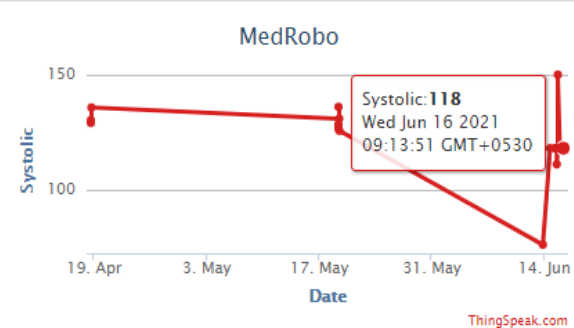

Fig 13. Parameter chart of systolic blood pressure

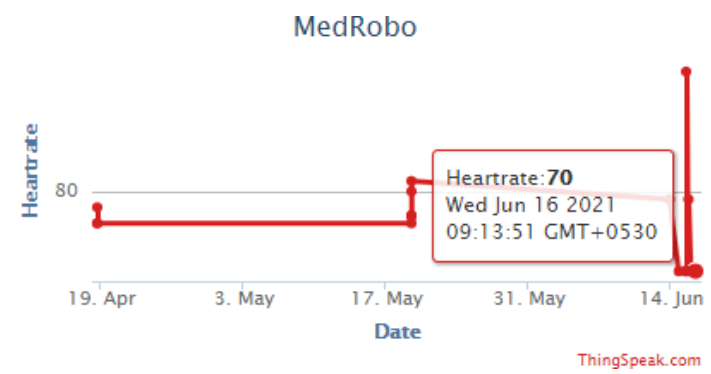

Fig 15 Parameter Chart of Heart Rate

\section{CONCLUSION}

We propose MedRobo to deliver medicine to the patients and check the patient vitals which alternate the nursing services. Since we need more number of input pins for our project implementation on the arduino board, arduino mega is preferred over other boards. The robot finds the path of movement and identifies the patient location using a line follower and with a RFID tag. This research helps to reduce human to human contact in hospitals and thus prevents the doctors and medical staff from getting affected by Coronavirus. The time of both patients and doctors is saved.

\section{REFERENCES}

[1]. Ankit R. Patel, Rajesh S. Patel, Navdeep M. Singh and Faruk S. Kazi, "Vitality of Robotics in Healthcare Industry - An Internet of Things (IoT) Perspective," Springer International Publishing AG 2017.

[2]. McNickle, M, "Medical Robots that could change Healthcare" In: IEEE International Conference Robotics and Automation (ICRA), Anchorage, 2010, 11 pp. p 300-307, 2012

[3]. Wall, J.,Chandra, V., Krummel, T., "Robotics in General Surgery, Medical Robotics. In Tech Publications, p 12. European Commission, Information Society, Brussels, 2008.

[4]. Ankur Roy Chowdhury, "IoT and Robotics: A Synergy" CC BY 4.0 Open Access rec: 31 Jan 2017, publ: 31 Jan 2017.

[5]. E. Guizzo, E. Ackerman, "The rise of the robot worker," IEEE Spect. 49 (10), 2012.

[6]. James J. Kuffner, "Cloud-Enabled Robots,” In IEEE-RAS International Conference onHumanoid Robots, Nashville, TN, 2010.

[7]. Rajesh. K, M. GopiKrishna, V.R.Rao, P.Pavani, C.Chandrasekhara "Smart Applications using Robotic and Iot Technologies in Fighting against Pandemic Covid19 in Medical and Societal Sectors," International Journal of Innovative Technology and Exploring Engineering (IJITEE) ISSN. 2278-3075, Volume-9 Issue 7 , 2020.

[8]. Min-soo Kang,Chunhwa Ihm, Jaeyeon Lee, Eun-Hye Choi, Sang Kwang Lee, - “A Study on Object Recognition for Safe Operation of Hospital Logistics Robot Based on IoT, " The Journal of The Institute of Internet, Broadcasting and Communication (IIBC) Vol.17, No.2, pp.141-146,. pISSN 2289-0238, eISSN 2289-0246.Apr. 30, 2017

[9]. Dr.K.Lakshmi Narayanan , Dr.N.Muthukumaran , Dr.G.Rajakumar, "Design and Fabrication of Medicine Delivery Robots for Hospitals.'International Conference Trends in Computing, Communication and Networking Technologies (ICRTCCNT'19), Kings Engineering College, October 18-19, 2019.

[10]. Seohyun Jeon, Jaeyeon Lee, "Performance analysis of scheduling multiple robots for hospital logistics" in the proceedings of 14th International Conference on Ubiquitous Robots and Ambient Intelligence (URAI) 2017 


\section{International Advanced Research Journal in Science, Engineering and Technology}

Vol. 8, Issue 6, June 2021

DOI: $10.17148 /$ IARJSET.2021.8679

[11]. Huiru Cao, Xiaofeng Huang, Jianyi Zhuang, Jianqiang Xu, Zening Shao Nanfang,

[12]. “CIoT-Robot: Cloud \& IoT Assisted Indoor Robot for Medicine Delivery” Joint International Advanced Engineering and Technology Res 2018

[13]. Torkestani S S, Julien-Vergonjanne A, Cances J, "Indoor optical wireless system dedicated to healthcare application in hospital, " International Symposium on Communication Systems Networks and Digital Signal Processing. IEEE, 2010.

[14]. Yu C, Chen X, "Home monitoring system based on indoor service robot and wireless sensor network," Computers \& Electrical Engineering, 39(4):1276-1287. -2013

[15]. Nimit Sheth, Chirag Sharma, Abhishek Sanil, Sahil Jethwa, "Robotic Assistance and Patient Monitoring in Hospitals using IoT"International Research Journal of Engineering and Technology (IRJET) Volume: 06 Issue:05, 2019.

[16]. Grieco, L.A, A. Rizzo, S. Colucci, S. Sicari, G. Piro, D. Di Paola and G. Boggia, "IoT-aided robotics applications: Technological implications, target domains and open issues," Computer Communications 54 ,32-47 Web, 2014.

[17]. Diksha B. Wasankar, Dr. Vijay S.Gulhane, L. K. Gautam, “Application of Internet of Things in the Field of Medical and Smart Health Care," International Journal of Innovative Research in Computer and Communication Engineering ,An ISO 3297, 2007 Certified Organization.

[18]. Ramanathan S; Akash V; Arun Prasad R; Venkat Rahul M. "Pick and Place Robot for Surface Mounting Devices". International Research Journal on Advanced Science Hub, 2, 8, 2020, 75-81. doi: 10.47392/irjash.2020.97

[19]. Gayathri N Shenoy; Chithu Rajan; Shibi Varghese; Vignesh M.V; ShanmugaPriya M; Priya S; Aparna George. "STERILOID: Room Sanitization Robot". International Research Journal on Advanced Science Hub, 2, 8, 2020, 100-104. doi: 10.47392/irjash.2020.101

[20]. Karthikeyan A.G.; Kishan K; Pattabiraman M; Prathiv S. "Medical Assistive Robot (MAR)". International Research Journal on Advanced Science Hub, 2, Special Issue ICAMET 10S, 2020, 71-75. doi: 10.47392/irjash.2020.201

[21]. Kaaviya L. "Design, analysis and data management of open Drain/Sewer solid waste cleaning robot". International Research Journal on Advanced Science Hub, 2, Special Issue ICSTM 12S, 2020, 88-92. doi: 10.47392/irjash.2020.267 